\title{
Ion Soft-Landing into Liquids: Protein Identification, Separation, and Purification with Retention of Biological Activity
}

\author{
Bogdan Gologan, Zoltán Takáts, Jormarie Alvarez, Justin M. Wiseman, \\ Nari Talaty, Zheng Ouyang, and R. Graham Cooks \\ Department of Chemistry, Purdue University, West Lafayette, Indiana, USA
}

Protein ions, after mass spectrometric separation, can be soft-landed into liquid surfaces with preservation of their native structures. Retention of biological activity is strongly favored in glycerol-based surfaces but not in self-assembled monolayer solid surfaces. Soft-landing efficiency for multiply-charged hexokinase ions was found to be some four times higher for a glycerol/fructose liquid surface than for a fluorinated self-assembled monolayer surface. Soft-landing into liquid surfaces is also shown to allow (1) protein purification, (2) on-surface identification of the soft-landed material using MALDI, and (3) protein identification by in-surface tryptic digestion. Pure lysozyme was successfully isolated from different mixtures including an oxidized, partially decomposed batch of the protein and a partial tryptic digest. Liquid glycerol/carbohydrate mixtures could be used directly to record MALDI spectra on the soft-landed compounds provided they were fortified in advance with traditional MALDI matrices such as p-nitroaniline and $\alpha$-cyano-4-hydroxycinnamic acid. Various proteins were soft-landed and detected on-target using these types of liquid surface. Soft-landing of multiply-charged lysozyme ions onto fluorinated self-assembled monolayer surfaces was found to occur with a limited amount of neutralization, and trapped multiply-charged ions could be desorbed from the surface by laser desorption. Initial data is shown for a new approach to protein identification that combines top-down and bottom-up approaches by utilizing protein ion soft-landing from a protein mixture, followed by tryptic digestion of the landed material and detection of characteristic tryptic fragments by MALDI. (J Am Soc Mass Spectrom 2004, 15, 1874-1884) (C) 2004 American Society for Mass Spectrometry

$\mathrm{M}$ olecular ion soft-landing, characterized by deposition of intact molecules on surfaces at low kinetic energies [1], has been applied in experiments ranging from studies using chemically inert and structurally organized self-assembled monolayers (SAMs) as substrates for ion storage [2], to studies of ion mobility through ice and vapor deposited films [3, 4], to experiments aimed at developing a separation method in a form of preparatory mass spectrometry [5, 6]. Simple organic cations [7, 8], metal clusters [9], polysaccharides [10], a nucleotide [11], and intact viruses [12-14] have all been the targets of examination by ion soft-landing. The separation, purification, and storage of chemical species by a completely non-destructive, mass spectrometric approach are the objectives of the studies currently underway in our laboratory.

In a preliminary report [5], we described a method which showed the potential for generating biologically

Published online November 17, 2004

Address reprint requests to Dr. R. G. Cooks, Department of Chemistry, Purdue University, West Lafayette, IN 47907-1393, USA. E-mail: cooks@ purdue.edu active protein chips from protein mixtures by massselective ion soft-landing. After ionization of the mixture by electrospray (ESI), individual protein ions were mass-selected and soft-landed at predetermined locations on the surface. The results showed that the use of mass spectrometry as a separation method in biology provides high selectivity because components with different molecular formulas can be separated and softlanded. However, retention of the biological activity of a protein or other biomolecule in the course of this (or any mass spectrometry experiment) is a much more challenging problem. This is the subject addressed in the present paper through the combined choice of gentle ionization methods and an appropriate liquid surface for soft-landing.

It is well known that polyols and sugars can efficiently stabilize a protein in an otherwise denaturating environment $[15,16]$, although the underlying mechanism of this process has not been elucidated. Lakshmi and Nandi [17] in 1976 showed that sucrose and glucose decrease the solubility of phenylalanine, tyrosine, and tryptophan in aqueous solution and suggested that this was due to increased hydrophobic interactions. Another notable study [18] investigated the effect of sor- 
bitol on the structure of hen egg white lysozyme by nuclear magnetic resonance and pointed out the small but significant changes in protein structure and dynamics that occur upon sorbitol addition, such as motional restriction of some alanine and threonine methyl groups, the appearance of new intramolecular nuclearOverhauser effect cross-peaks in the hydrophobic core, and changes in protein hydration. These data suggest that proteins, in the presence of polyol based additives, will experience reduced local motions and partial compression of the entire structure which may explain the reduced level of denaturation under the influence of temperature and $\mathrm{pH}$ stress. Moreover, prior reports demonstrated that polyols, in combination with sugars, have a positive effect on the refolding and reactivation of guanidium chloride-denaturated arginine kinase [19]. Overall, polyol-based liquid environments, for example sucrose or fructose mixtures with glycerol, have proven beneficial in stabilizing proteins through freezing point depression, conformational effects, preferential hydration, and domain interface changes. Reduction in backbone mobility and its underlying role as a bacteriostatic agent make glycerol the medium of choice for short- and long-term storage of valuable proteins and peptides. Given these considerations and the fact that separation and collection of proteins by mass spectrometry, through the use of ion soft-landing has already been demonstrated [5], a logical step is to attempt to separate proteins and peptides using glycerol and glycerol-based surfaces as landing substrates and to establish whether biological activity could be maintained though the entire process of ionization, mass analysis, soft-landing, and storage in the liquid medium.

The family of desorption ionization (DI) techniques, including liquid secondary ion mass spectrometry (LSIMS) [20], fast atom bombardment (FAB) [21-25], and matrix-assisted laser desorption ionization (MALDI), provide one type of approach to the examination of the post soft-landed materials. In early reports, Hillenkamp et al. [26] and Tanaka and coworkers [27] used liquid matrices as means to detect various analytes by mass spectrometry. While solid organic chromophores are currently more widely used, there are disadvantages to solid state ionization, including highly variable desorption efficiency across the sampled surface, high dependence of the MALDI ion signal on the preparation technique, and difficulty in obtaining quantitative results for experiments which require a sustainable stable signal intensity over a long time span. On the other hand, liquid MALDI matrices can be made to retain many of the advantages of solid matrices and, in addition, also provide a homogeneous sample, consistent ion signal intensity for a large number of shots directed at the same spot, reduced desorption energies and the opportunity to control sample chemistry and so enhance ionization [28, 29]. A less desirable alternative to DI analysis of the soft landed materials is the use of electrospray ionization (ESI). Unfortunately, analysis of proteins, using ESI, after ion soft-landing into solutions containing high concentrations of glycerol introduces new problems, mainly in the detection of the landed analytes. The low vapor pressure of glycerol and its high affinity for proteins create interferences due to adduct formation, clustering, and supercharging effects [30-32]. On a positive side, most of these disadvantages can be overcome though the use of an ESI variant, electrosonic spray ionization (ESSI) [33]. This method can be considered a viable alternative to desorption ionization based analysis of soft landed materials when instruments capable of L-SIMS or MALDI-TOF are not available.

The arguments made above indicate that there is a need and an opportunity to develop liquid surfaces for ion soft-landing experiments. Desirable properties of liquids suitable for this type of application are (1) the liquids should be relatively nonvolatile, (2) they should rapidly and effectively solvate the arriving gas phase ions, and (3) they should facilitate gentle landing through effective intermolecular energy transfer. It is also desirable that it be possible to add enzymes and other reagents to the liquid so that in-situ reactions with the soft landed analyte can be carried out.

In this paper, we demonstrate new capabilities for mass spectrometry by showing that it is possible to soft-land proteins and peptides from complex mixtures into glycerolbased liquid surfaces with preservation of bioactivity. We also show that appropriate liquid surfaces used without any additional treatment to detect the soft-landed material by electrospray ionization (ESI), electrosonic spray ionization (ESSI) [33], or matrix-assisted laser desorption ionization mass spectrometry (MALDI). The liquids can also be further processed to extract the purified protein. Importantly, by adding appropriate enzymes to the liquid surfaces used for soft-landing substrates, the nature of purified proteins can be determined through enzymatic reactions. For example, in the case of proteolytic enzymes, enzymatic fragmentation can be effected and followed by analysis of the resulting peptides by standard methods.

\section{Experimental}

\section{Materials}

Bradykinin, glycerol, lysozyme, p-nitroaniline, 2,5-dinitrobenzoic acid (DHB), 3-aminoquinoline, $\alpha$-cyano-4hydroxycinnamic acid (CHCA), sinapic acid (SA), ammonium bicarbonate, hydrogen peroxide, sulfuric acid, 11-mercaptoundecanol, and cytochrome $c$ were purchased from Sigma-Aldrich (St. Louis, MO). Fructose and sucrose were purchased from Fisher Scientific (Hanover Park, IL). Trypsin was purchased from Worthington Biochemical Corporation (Lakewood, NJ). Hexa-N-acetylchitohexose was purchased from Seikagaku Corporation (Tokyo, Japan). All HPLC grade solvents such as methanol and water were obtained from Waters Corporation (Milford, MA). All of these chemicals were used without further purification. 


\section{Methods}

Commercially available gold substrates $(20 \mathrm{~mm} \times 50$ $\mathrm{mm}$ ) were acquired from International Wafer Service). They consisted of a Si wafer with $5 \mathrm{~nm}$ chromium adhesion layer and $200 \mathrm{~nm}$ of polycrystalline vapor deposited gold. Prior to soft-landing experiments, the substrates were cut into $2 \times 2 \mathrm{~mm}^{2}$ surfaces, cleaned in an acid bath containing 1:4 $\mathrm{H}_{2} \mathrm{O}_{2} / \mathrm{H}_{2} \mathrm{SO}_{4}$ and then washed thoroughly with deionized water and absolute ethanol. Self-assembled monolayers were formed by immersing the clean substrates into 11-mercaptoundecanol (OH-SAM) monomer solutions or 1,2-tetrahydroperfluorodecane disulfide $\left[\left(\mathrm{CF}_{3}\left\{\mathrm{CF}_{2}\right\}_{7}\left\{\mathrm{CH}_{2}\right\}_{2} \mathrm{~S}\right)_{2}\right](\mathrm{F}-$ SAM), prepared in ethanol $(1 \mathrm{mM})$. The self-assembly process was allowed to proceed over a period of 2 weeks.

Liquid surfaces were prepared by dissolving $\mathrm{p}$ nitroaniline, 2,5-dinitrobenzoic acid (DHB), $\alpha$-cyano-4hydroxycinnamic acid (CHCA) or sinapic acid (SA) in glycerol, glycerol:fructose (2:1) or glycerol:sucrose (2:1). The amount of DHB, CHCA and SA that could be dissolved in glycerol was increased by using the base 3-aminoquinoline as solubilizing agent [34-36]. The binary or tertiary mixtures formed through matrix solvation in glycerol were further diluted with ethyl alcohol (15:1000 vol/vol), which acted both as a solvent and as a liquid surface delivery agent and allowed control of the thickness of the final liquid surface on the OH-SAM substrates. Evaporation of volatile solvent from the sample plate was assisted either by a stream of air or by vacuum.

For the experiments involving evaluation of bioactivity and soft-landing efficiency, the soft-landed hexokinase was rinsed from the surface using $15 \mu \mathrm{L} 10 \mathrm{mM}$ aqueous ammonium acetate. The quantity of hexokinase landed was measured by analyzing a $5 \mu \mathrm{L}$ aliquot of this rinse solution using a Thermo Finnigan (San Jose, CA) LCQ Classic instrument equipped with a home built ESSI ion source. The protein concentration was determined using loop injection together with external standard calibration using pure hexokinase solutions. The specific activity of hexokinase was determined by assaying the phosphorylating activity of the soft-landed enzyme using fructose as substrate. A $5 \mu \mathrm{L}$ aliquot of the surface rinse solution was mixed with $5 \mu \mathrm{L}$ of a solution containing $1 \mathrm{mM}$ ATP $\mathrm{Mg}$ salt and $10 \mathrm{mM}$ ammonium acetate at $\mathrm{pH}$ 7.8. In the case of the F-SAM surface, the rinse solution contained the exact amount of liquid surface material (glycerol/fructose) which was used in the parallel liquid surface landing experiment. The mixture was incubated at $30^{\circ} \mathrm{C}$ for $30 \mathrm{~min}$. The concentration of active enzyme was determined by measuring the concentration of fructose-6-phosphate using negative ion ESI-MS/MS for the whole assay procedure. The ion at $\mathrm{m} / \mathrm{z} 259$ (deprotonated molecular ion of fructose-6-phosphate) was isolated and fragmented at $15 \%$ relative dissociation energy. Fragment ions at $\mathrm{m} / \mathrm{z} 79$ and 97 were monitored in an MS/MS experiment and their abundances were used for quantification in a reaction monitoring MS/MS experiment. Calibration was achieved by adding different amounts of enzyme to similar quantities of liquid surfaces and repeating the process described above. All samples were analyzed in a flow-injection mode. The specific activity was obtained by comparing the total concentration of hexokinase to the concentration of active enzyme and the relative standard deviation of the results was between 10 and $20 \%$.

Tests of bioactivity of the enzyme-doped liquid surfaces were performed using a surface composed of L-1-chloro-3-[4-tosylamido]-4-phenyl-2-butanone (TPCK) modified trypsin and glycerol, immobilized on an OH-SAM/Au support. An estimated 1 to $10 \mathrm{ng}$ of bovine cytochrome $c$ was soft-landed over a period of $75 \mathrm{~min}$ from a solution containing $0.1 \mathrm{mg} / \mathrm{ml}$ cytochrome $c$ and $0.01 \mathrm{mg} / \mathrm{ml}$ lysozyme. Except where otherwise mentioned, soft-landing was done by mass selecting the ion $(\mathrm{M}+15 \mathrm{H})^{+15}$ using an $m / z 15$ window. Upon recovery of the liquid surface into which the cytochrome $c$ had been landed, digestion was initiated by adding $50 \mu \mathrm{l}$ of ammonium bicarbonate $(0.01 \mathrm{M})$. The reaction mixture was incubated at constant $37^{\circ} \mathrm{C}$ overnight in parallel with a blank sample that did not contain any cytochrome $c$.

\section{Instrumentation}

The soft-landing of mass-selected multiply-charged ions was achieved using a single-stage quadrupole model SSQ-710C (Thermo Finnigan, Bremen, Germany) or by using a home-built linear ion trap mass spectrometer (SL1.0) [37]. The SSQ instrument (Figure 1a) was modified to allow a surface to be positioned in the vacuum chamber. The modification consisted of a moving stage that included a rotating disk to which the surface was attached at a predetermined location. The surface was positioned between the quadrupole and the detector. The rotating disk included an aperture that could be used to allow the ions to pass directly to the detector when desired. Rotation was controlled using a stepper motor. The functionalized gold surfaces were attached to the disk with conductive copper tape with adhesive on both surfaces.

Ionization of protein solutions employed a homebuilt electrospray ionization (ESI) source. A voltage of 4 $\mathrm{kV}$ was applied to the syringe tip while the flow rate of the solution was set at $0.3 \mu \mathrm{l} / \mathrm{min}$. The temperature of the heated capillary was set to $180{ }^{\circ} \mathrm{C}$ and the instrument was prepared for acquisition of mass spectra to characterize the protein solution. In this mode, later referred to as the ion detection mode, the high voltages on the conversion dynode and the multiplier were turned on, the ions were detected and overall spectral quality, including signal-to-noise ratio and mass resolution over the full mass range (up to $2000 \mathrm{Th}$ ), was examined. Next, the rotating disk was positioned so that the surface mounted on the disk was located in-line 


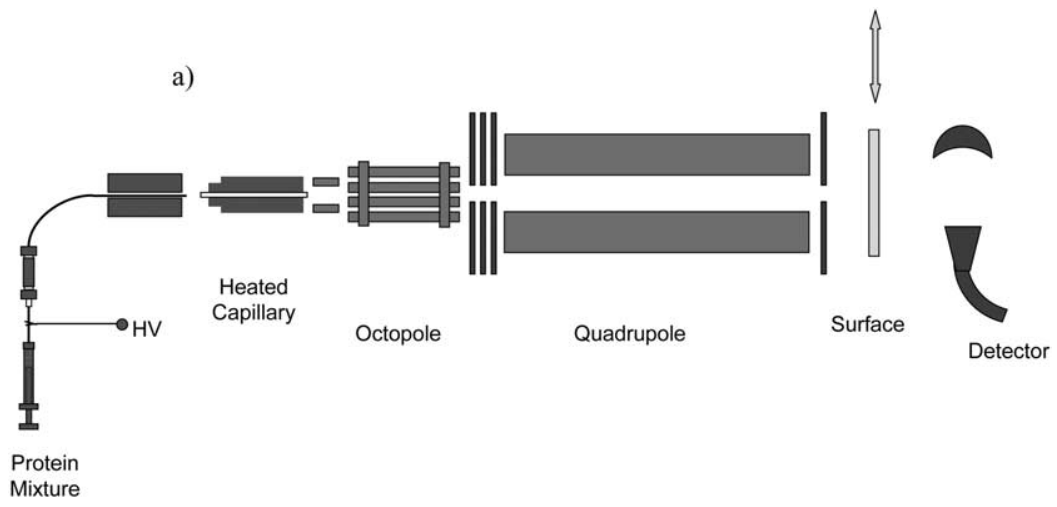

b)

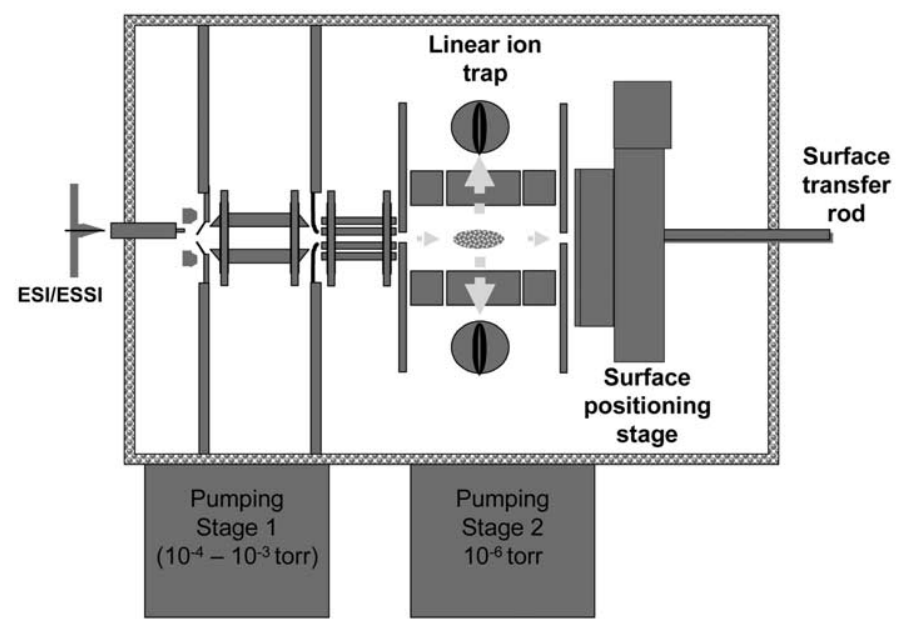

Figure 1. (a) Schematic representation of the ThermoFinnigan SSQ 710c instrument as modified and used for ion soft-landing; (b) Top view: schematic of the prototype linear ion trap instrument built for ion soft-landing and its surface loading mechanism. Note that the image depicts ions being ejected radially from the trap along the $\mathrm{x}$-axis for detection and that the surface is aligned along the $\mathrm{z}$-axis (y-axis is coming out of the plane of the page). The depiction shows the ESI source, heated capillary atmospheric interface, tube lens, skimmer, square quadrupole ion guide, inter-multipole gate lens, and octopole ion guide as well as the other labeled components.

with the ion beam and at the exit of the quadrupole mass filter. In this mode, subsequently referred to as the ion-landing mode, the voltages on the conversion dynode and the multiplier were turned off and the ions were allowed to impinge on the surface. The voltage on the surface was set to $0 \mathrm{~V}$ giving a nominal value of zero as the collision energy used in the landing process. The total ion dose used in each of the soft-landing experiments was on the order of $10^{10}-10^{11}$ ions as estimated by taking into account the intensity of the mass-selected ion beam at the electron multiplier, the voltages applied on the conversion dynode and the electron multiplier, the corresponding calibration factors for the detection system, and the total ion soft-landing time.

A diagram of the linear trap instrument (LIT) is shown in Figure $1 \mathrm{~b}$ and its detailed operation is described elsewhere [37]. This instrument includes an ESI ion source, a high-capacity LIT, wave form capabilities for ion isolation, a radial detector for mass analysis, and facilities for low energy transfer of trapped, mass- selected ions onto specific spots onto an electronically movable target. In comparison with the SSQ instrument, the linear ion trap instrument was customdesigned and built for soft-landing and it has a number of advantages in terms of ease of use, control of spot positions in arrays, and flexibility in experimental design.

Soft-landed material was analyzed by using ESI-MS or ESSI-MS [33] on an LCQ Classic (ThermoFinnigan, Bremen, Germany) or by using MALDI-MS on a Bruker Reflex III MALDI-TOF instrument. Except where otherwise stated, the surface material recovered after softlanding was used without additional treatment. The MALDI-TOF was equipped with a nitrogen laser (337 $\mathrm{nm}$ ) and operated at an acceleration voltage of $25 \mathrm{kV}$. The laser data was collected in reflectron mode in the low mass range, and in the linear mode for the high mass range. A MALDI mass spectrum consisted of the average ion signal acquired from 100 to 200 shots, with good shot-to-shot reproducibility due to the homogene- 
ity in the matrix-containing liquid surface. In the case of analysis by ESI-MS, the proteins or peptides separated by ion soft-landing were recovered along with the liquid surface by washing the gold support with $50 \mu \mathrm{l}$ of a solution of 50:49:1 methanol:water:TFA ( $\mathrm{vol} / \mathrm{vol} /$ vol).

\section{Results and Discussion}

Ion soft-landing is a relatively new separation and preparation method that is applicable to biological molecules, including proteins [5]. It can be used to identify proteins and purify them from complex mixtures. The present study follows up on a preliminary report [5] of these capabilities but it also raises the more difficult question: "Can the bioactivity of soft-landed proteins be preserved?" The answer is in the affirmative, at least for the selected proteins examined by ion soft-landing into appropriate liquid surfaces.

\section{Isolation of Proteins from Complex Mixtures by Ion Soft-Landing}

A typical result from an experiment that tests the purification efficiency of a crude protein sample by ion soft-landing into a liquid surface is shown in Figure 2. In this experiment, lysozyme from an oxidized and partially decomposed sample was ionized using conventional ESI and, based on a preliminary mass spectral analysis (Figure 2a), ions corresponding to the +10 charge state $(1430-1435 \mathrm{Th})$ were isolated using the modified SSQ instrument and collected into a glycerol/sucrose liquid matrix. Subsequent cleanup of the solution was used to remove the glycerol matrix which interferes in the ESI analysis by producing a supercharging effect [31,32]. The simplest method of matrix removal consisted of carrying out a solvent exchange using $3 \mathrm{kDa}$ ultrafilter membranes and ammonium acetate buffer $(\mathrm{pH}=$ 7.0). The result of mass spectrometric analysis performed on the liquid after soft-landing purification is shown in Figure $2 \mathrm{~b}$. The liquid matrix containing soft landed material was rinsed using $1 \mathrm{~mL}$ methanol/ water $1: 1$ and the rinse liquid was concentrated through ultrafiltration using a Microcon YM-3 centrifugal filter unit. The concentrate was diluted with methanol/water 1:1 10-fold, and when analyzed using the LCQ instrument under similar conditions to those used for obtaining the preliminary information about the impure sample, it produced the spectrum of pure lysozyme shown in Figure $2 b$. The nature of the impurities in the original sample was not identified. The results indicate that it is possible to softland pure proteins into a glycerol containing liquid surface starting with a complex protein mixture.
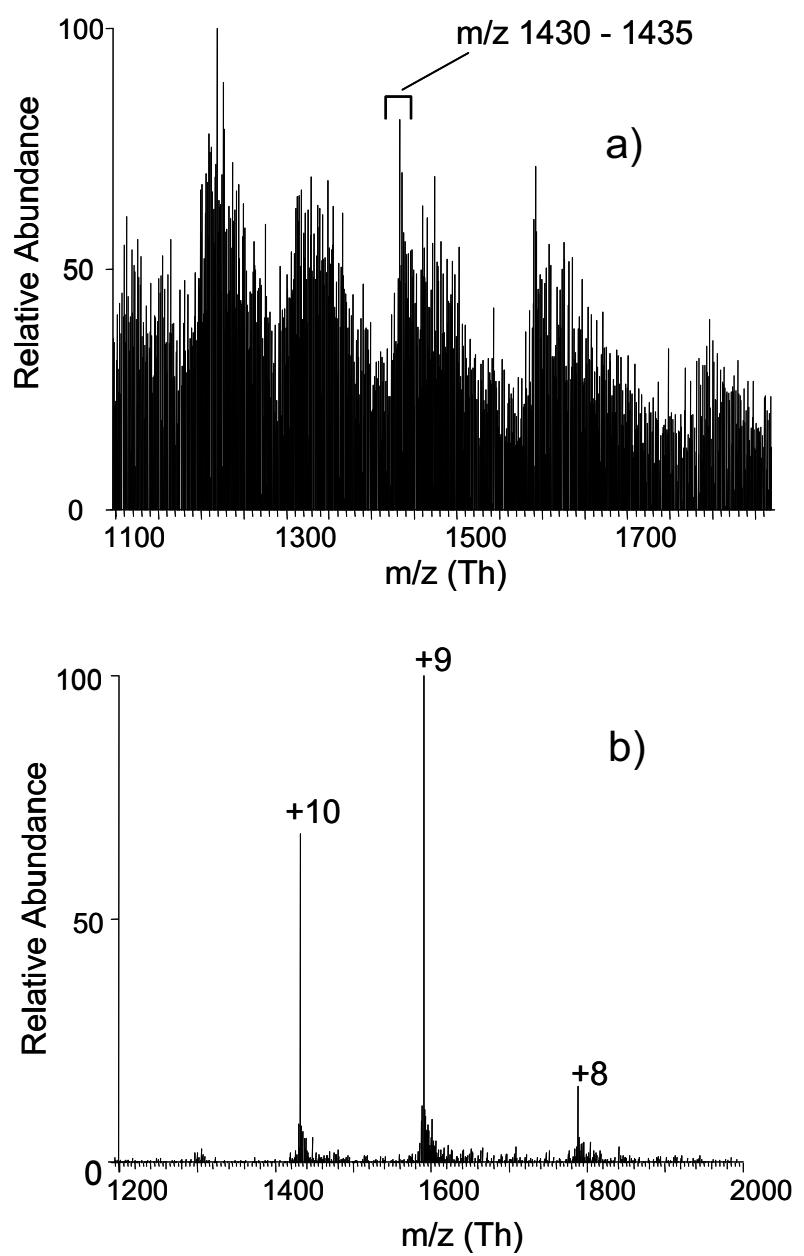

Figure 2. Comparison of the ESI mass spectrum of (a) an impure sample of lysozyme with that of (b) a purified sample after soft-landing ions of $m / z 1430-1435$ (only the +10 charge state) into a glycerol/sucrose liquid matrix. The landed material was separated from the matrix by ultrafiltration and shows an ESI mass spectrum that displays the lysozyme charge states $+10,+9$, and +8 consistent with neutralization upon landing.

\section{Bioactivity Preservation Upon Ion Soft-Landing}

As mentioned, one of the most desirable characteristics of ion soft-landing would be the ability to maintain the bioactivity of proteins that are collected after the gas phase separation and step. To test the hypothesis that enzymes can still function after removal from the aqueous environment, ionization, mass-analysis in vacuum and then resolvation, we utilized lysozyme as a test protein. Lysozyme is an enzyme with an antibacterial role because it degrades the polysaccharide component found in the cell walls of many bacteria. Lysozyme acts by catalyzing the insertion of a water molecule into the polysaccharide chain at the point of cleavage. Therefore, we chose to soft-land the protein into a liquid surface containing glycerol (with fructose added to increase its viscosity and decrease its vapor pressure) and hexa-N-acetylchitohexaose, a molecule composed of six $\mathrm{N}$-acetylglucosamine units, a common target for lysozyme. The ion [lysozyme $+8 \mathrm{H}]^{8+}$ was landed for 

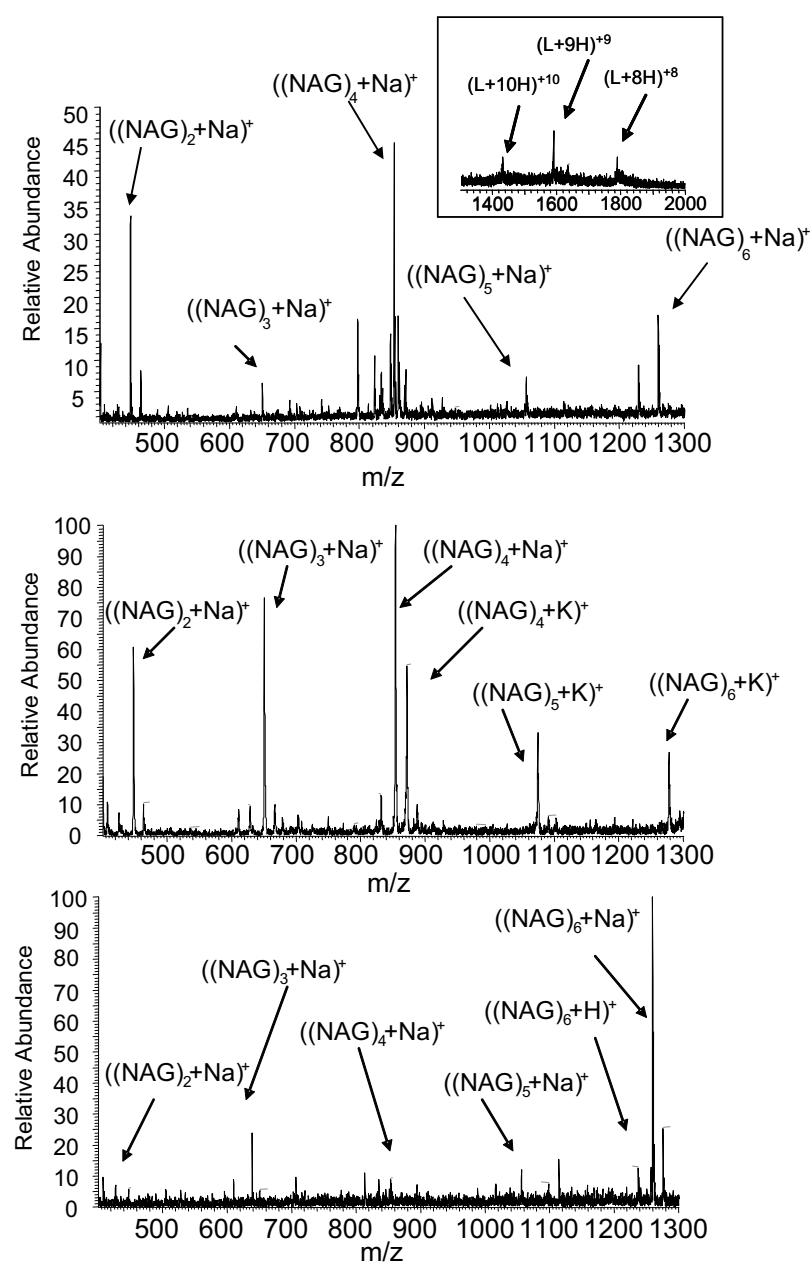

Figure 3. Comparison of N-acetylglucosamine hexamer hydrolysis under enzymatic and non-enzymatic conditions: (a) ESI-MS spectrum of an solution containing lysozyme after soft-landing the ion $(\mathrm{M}+8 \mathrm{H})^{+8}$ into a glycerol/fructose/hexa-N-acetylchitohexose liquid surface. The landed material, along with the supporting substrate, was recovered in a buffered solution, incubated, diluted with methanol, and injecting into an LCQ Classic for ESI analysis. The ESI mass spectrum displays the sodiated N-acetylglucosamine hexamer (NAG) ${ }_{6}$ as well as the sodiated N-acetylglucosamine pentamer $(\mathrm{NAG})_{5}$, tetramer $(\mathrm{NAG})_{4}$, trimer $(\mathrm{NAG})_{3}$, and dimer (NAG) $)_{2}$ resulting from lysozyme bioactivity. Soft-landed lysozyme (L) is also observed (see insert); (b) ESI-MS spectrum corresponding to hydrolysis of $\mathrm{NAG}_{6}$ by solution-phase lysozyme (Blank 1); (c) ESI-MS spectrum corresponding to $\mathrm{NAG}_{6}$ stored overnight at the same temperature, $\mathrm{pH}$ and in the same reaction medium as in case (a) and (b) except that no lysozyme was present.

$1 \mathrm{~h}$ into the liquid target. Upon recovery of the liquid surface, the enzymatic reaction was triggered by addition of $\mathrm{NaHCO}_{3}$ buffer, adjusted to a final $\mathrm{pH}$ of 6.5-7.5. The solution was incubated at $38^{\circ} \mathrm{C}$ and the reaction was allowed to proceed overnight. Upon dilution of the digestion medium and analysis by ESI-MS, the spectrum shown in Figure 3a was recorded. Close inspection reveals that the main digestion products, $\mathrm{N}$-acetylglucosamine pentamer, tetramer, trimer and dimer, expected upon reaction of lysozyme with hexa-N-acetylchitohexose, are all present in the reaction mixture along with the traces of soft-landed lysozyme. To further substantiate the findings, two additional samples were run in parallel with the incubation of the softlanded lysozyme. Those two samples contained, in one case, a control with identical composition as in the above reaction except that lysozyme was added from a standard solution rather than being deposited through soft-landing (Figure $3 b$ ). In the other, a blank, lysozyme was excluded from the reaction mixture so that we could study the effects of $\mathrm{N}$-acetylglucosamine hexamer self-hydrolysis. Overall, a distinct difference between the self-hydrolysis of $\mathrm{NAG}_{6}$ (Figure 3c) and hydrolysis of $\mathrm{NAG}_{6}$ by soft-landed lysozyme (Figure $3 \mathrm{a}$ ) was observed. Moreover we are able to confirm that the fragments corresponding to the digested sugar were due to the enzymatic activity of soft-landed lysozyme because the same fragment was observed when solution-phase lysozyme was added to a $\mathrm{NAG}_{6}$ liquid surface containing medium and allowed to react overnight (Figure 3b). A higher incidence of $\mathrm{Na}^{+}$and $\mathrm{K}^{+}$ adducts in the case of solution-phase lysozyme digestion (Figure $3 b$ ) is attributed to the impurities present in the commercial lysozyme samples, which can be eliminated through soft-landing purification.

\section{Efficiency of Soft-Landing for Different Substrates}

Mass spectrometric analysis of soft-landed materials in a glycerol-based liquid is not straightforward, since such materials are not readily compatible with either MALDI or ESI-MS. However, variants of ESI exist which are compatible with high glycerol concentrations. Systematic studies on hexokinase soft-landing employed a gentle electrospray variant, electrosonic spray ionization (ESSI) [33]. The analytical performance of ESSI, including sample consumption and sensitivity, includes the production of narrow peaks (little or no solvation or other adduct formation) and an extremely narrow charge-state distribution as exemplified by a spectrum of hexokinase shown in Figure 4. The method tends to provide ions of proteins and protein complexes in their native conformation rather than their denatured states [38].

In a systematic study of soft-landing experiments done at landing energies in the range of $0-10 \mathrm{eV} /$ charge, the landing efficiency and the specific bioactivity of yeast hexokinase were compared for the cases of a fluorinated self-assembled monolayer surface and a glycerol/fructose (3:2) surface. For both surfaces, the optimal landing efficiency, defined as recovered mate$\mathrm{rial} /$ number of ions directed at the surface, was observed to be ca. $3 \mathrm{eV} /$ charge. At an energy of 10 $\mathrm{eV} /$ charge the efficiency is an order of magnitude lower. The reasons for the decrease in soft-landing efficiency are not known but can reasonably be associated with the increasing likelihood of simple scattering at lower energy and surface-induced dissociation at higher energies [39]. Note that the pressure in the linear ion trap instrument is $\sim 1$ mtorr and that the distance 


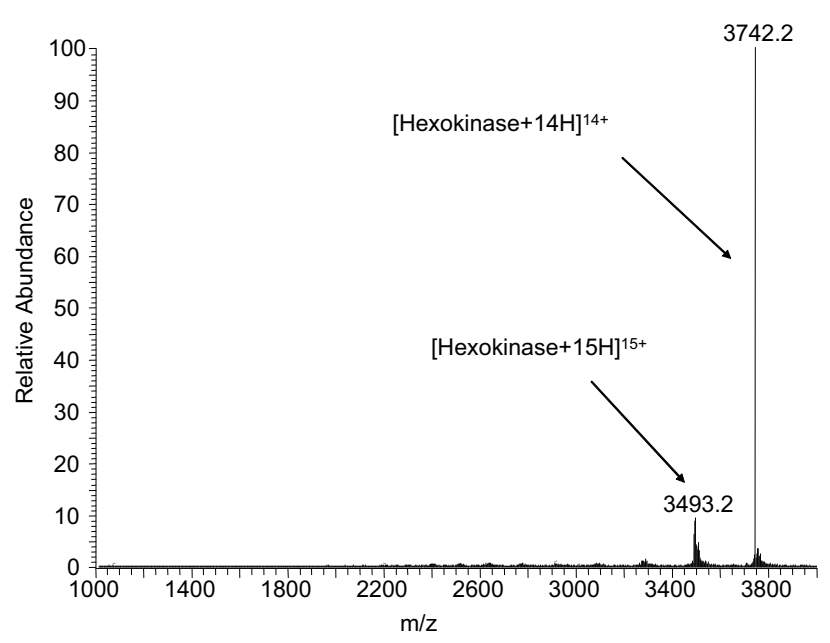

Figure 4. ESSI-MS spectrum of hexokinase in $10 \mathrm{mM}$ aqueous ammonium acetate solution buffered at $\mathrm{pH} 5$.

from the center of the trap to the surface is an order of magnitude greater than the mean free path of hexokinase ions under these conditions. Comparison of the soft-landing efficiency of hexokinase ions between the liquid surface and the fluorinated self-assembled monolayer substrate showed a five-fold increase in efficiency throughout the energy range examined for the liquid substrate. Not only was the ion soft-landing efficiency higher on this surface but the specific activity of the soft-landed proteins was also correspondingly higher in the case of the liquid than the solid self-assembled monolayer surface. The specific activity of the landed hexokinase was determined using an assay that measured the phosphorylating activity of the soft-landed enzyme using fructose as the substrate. A similar assay has been reported by Leary and coworkers [40] and we have previously used glucose as substrate in related assays [5]. More specifically, the soft-landed hexokinase was recovered from the surface by rinsing with ammonium acetate buffer and an aliquot of this solution was buffered and ATP Mg salt was added. In the case of the F-SAM surface, the rinse solution was enriched with glycerol to reproduce the liquid surface material (glycerol/fructose) which was used in the parallel liquid surface landing experiment. Subsequent to incubation, the concentration of active enzyme was determined by measuring the concentration of fructose-6-phosphate using negative ion ESI-MS/MS, monitoring the loss of $\mathrm{PO}_{3}$ from the deprotonated fructose monophosphate [5]. External calibration of the assay employed standard hexokinase solutions containing 0.1 to $10 \mathrm{ng}$ of pure enzyme. The specific activity was obtained by comparing the total amount of hexokinase in the rinse solution to the amount of active enzyme. Even within the optimum landing energy range of $3 \mathrm{eV} /$ charge, the bioactivity of the recovered hexokinase was very small for the case of the fluorinated self-assembled monolayer functionalized surface and approximately quantitative in the liquid surface experiment. The specific activity

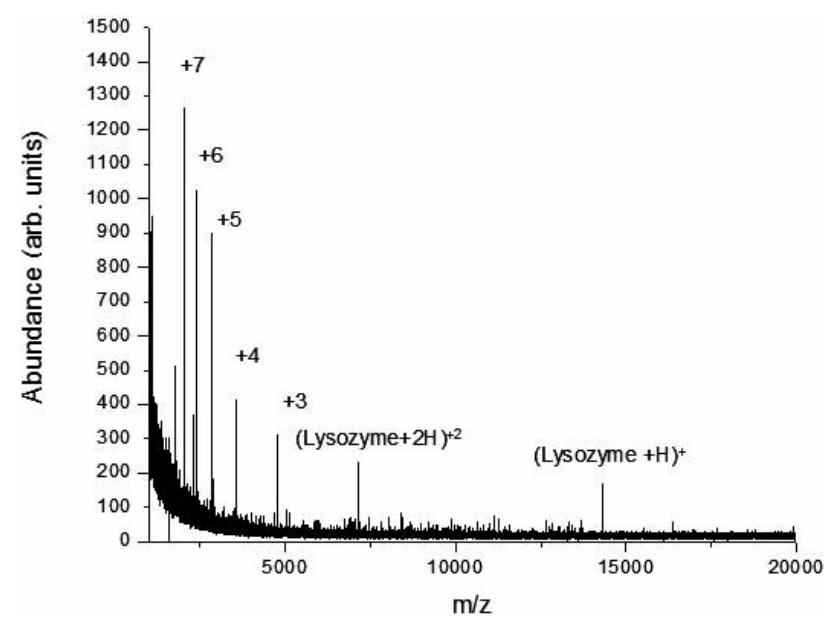

Figure 5. LDI-MS spectrum of soft-landed lysozyme $(\mathrm{M}+8 \mathrm{H})^{+8}$ on a $\mathrm{CF}_{3}$-terminated SAM surface.

decreased rapidly for both surfaces when the experiment was performed outside the optimum deposition energy range and in the F-SAM case no signal for fructose-6-phosphate could be observed above $5 \mathrm{eV} /$ charge.

The difference in landing efficiency and residual bioactivity can be explained by considering events during the landing process. In the case of the fluorinated F-SAM surface colliding ions most likely undergo some conformational reorientation, in some cases unfolding, to expose the hydrophobic core to the substrate. However, since the protein ions most likely do not unfold completely, some of the charges still can remain isolated from the hydrophilic surface groups causing surface charge build-up. Hence, subsequent protein ions experience the effect of a net charge on the surface and an insulating layer of proteins, factors that could contribute to the observed low soft-landing efficiency. In the case of the liquid surface, the approaching protein ions undergo a series of low energy collisions with evaporating glycerol molecules gently removing excess kinetic energy. If some of the protein charge sites are still isolated and the solvent molecules are able to provide counter ions to yield a neutral surface. Beyond these advantages features, glycerol is well known to stabilize folded protein structures [15].

The assumed incomplete neutralization on the FSAM surface implies that the landed ions can be desorbed from a positively-charged surface without any need for a supplementary matrix with a strong absorption band in the UV. In agreement with this expectation, laser desorption spectra of soft-landed lysozyme on an F-SAM surface shows multiply-charged ions corresponding to several protonated charge states of the landed species (Figure 5). Note that all the observed ions have lower charge states than the mass-selected soft landed ion itself, providing evidence for partial neutralization occurring on the surface. In related work from another laboratory, SIMS interrogation of F-SAM surfaces carrying soft-landed doubly-protonated brady- 

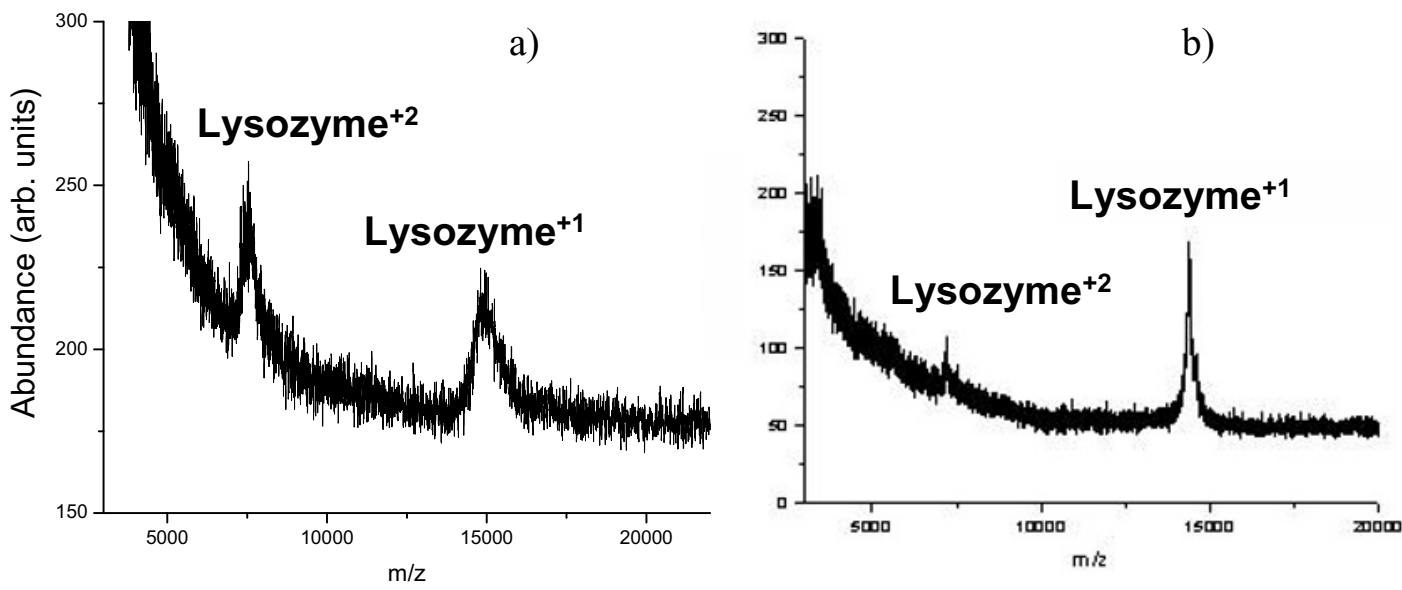

Figure 6. (a) UV-MALDI mass spectrum of a surface after soft-landing the ion [lysozyme $+9 \mathrm{H}]^{9+}$ into a glycerol/fructose/p-nitroaniline/water liquid. (b) UV-MALDI mass spectrum after softlanding [lysozyme $+9 \mathrm{H}]^{9+}$ into an $\alpha$-cyano-4-hydroxycinnamic acid:3-aminoquinoline:glycerol liquid mixture. No additional sample processing was used between soft-landing and MALDI analysis in either case. p-Nitroaniline in the liquid surface functioned as the UV-MALDI matrix in (a) and $\alpha$-cyano-4-hydroxycinnamic acid dissolved in the liquid surface functioned as a UV-MALDI matrix in (b).

kinin yielded doubly-charged ions of the peptide [41]. These observations on charge retention by landed ions at particular surfaces are in agreement with the results of early soft-landing experiments [2] and extend the previously observed phenomenon on ionic deposition on a surface to macromolecular systems. This result should allow further exploration into the preparation of surfaces with unusual electronic and biological properties.

\section{On-Target Analysis of Soft-Landed Materials}

In contrast to spray-based methods of analysis of soft-landed materials, which necessarily involve dissolution of the surface matrix, MALDI offers a type of analysis which can be carried out in principle without any further modification of the surface. Glycerol and carbohydrates themselves are not good matrices for UV-MALDI applications; however, addition of various UV absorbing chromophores (including traditional MALDI matrices) produces adequate liquid MALDI matrices and soft-landing can be performed directly into these matrices [42]. Soft-landing followed by MALDI detection using $\alpha$-cyano-4-hydroxycinnamic acid and p-nitroaniline doped glycerol surfaces gave the results shown in Figure 6. In these experiments the surface carrying the liquid matrix and the soft-landed material was directly transferred from the soft-landing instrument to the MALDI-TOF and the sample was interrogated using MALDI without any prior modification. The detected peaks are considerably broader than in the case of solid matrices, which is in agreement with the findings of previous studies [43]. In contrast to the undesired peak broadening, liquid matrices show a number of desirable features including good shot-to- shot spectral reproducibility and at least two orders of magnitude dynamic range, features which should allow quantification of the landed material using external calibration. Adequate spectral reproducibility can be associated with the homogenous distribution of constituents and analytes in the liquid matrix, features that cannot be achieved using a microcrystalline solid matrix. One of the most important implications of these experiments is the opportunity to perform soft-landing and analysis in the same device, viz. the possibility of doing in-situ analysis after ion soft-landing. Although the quadrupole analyzer is not compatible with MALDI ionization, since these analyzers only work with continuous ion sources, the linear ion trap (vide supra) will be combined with a MALDI source in such a way that the soft-landed material can be re-ionized and focused back into the linear ion trap. Since the sample consumption of MALDI analysis is negligible compared to the overall amount of analyte, this method might be used continuous throughout the soft-landing experiment to follow the progress of the experiment.

\section{In-Situ Digestion}

Liquid surfaces provide the capability of accumulating compounds delivered as mass-selected ions to the surface. This can be done in-situ using specific chemical and enzymatic reactions or aliquots of liquid surface material can taken and tested using various reactants to provide more specific information about the landed species. A special case of this type of application is when an active enzyme is dissolved in the liquid surface, and the landed substrate undergoes in situ enzymatic modification. As a demonstration, cytochrome $c$ ions were landed on a liquid surface which 


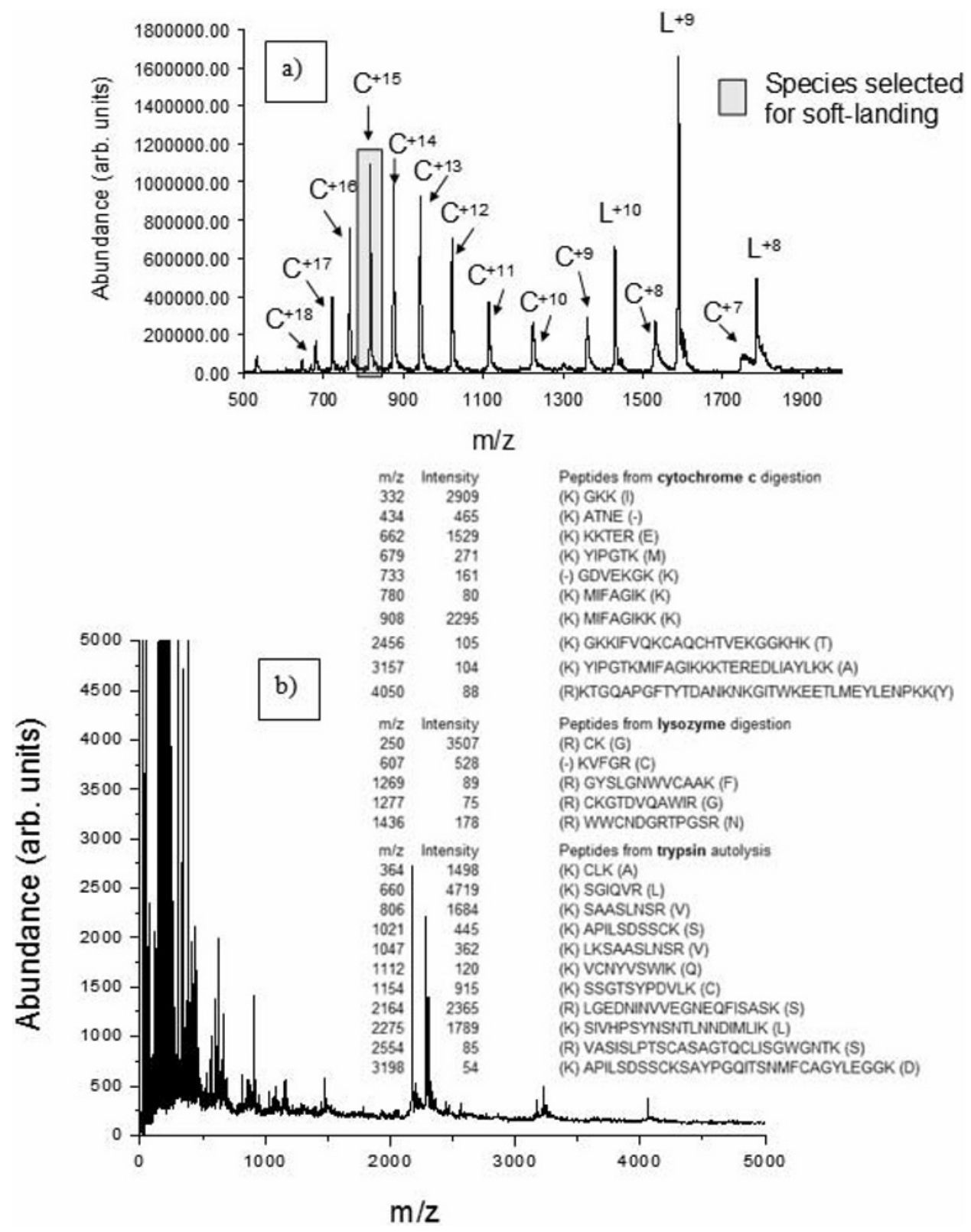

Figure 7. (a) ESI-MS spectrum of the lysozyme/cytochrome $c$ binary mixture used for the soft-landing experiment; (b)UV-MALDI-MS spectrum after cytochrome $c$ digestion in an enzymatically active liquid surface containing trypsin. The peaks identified in the insert are characteristic of peptides resulting from tryptic digestion of soft-landed cytochrome $c$ in a glycerol environment as well as peptides resulting from trypsin autolysis and lysozyme digestion.

contained active trypsin dissolved in a glycerol/fructose/water mixture. The cytochrome $c$ solution used for ion soft-landing contained an equal amount of lysozyme in order to represent results for a mixture. The cytochrome $c(\mathrm{M}+15 \mathrm{H})^{+15}$ ion with $\mathrm{m} / \mathrm{z} 825$ was isolated for soft-landing using an window of 15 units. After soft-landing, the surface material was diluted, incubated and $\alpha$-cyano-4-hydroxycinnamic acid was added as a matrix compound prior to MALDI analysis. Note, that from our experience all the tested MALDI matrices are strong inhibitors of trypsin at appropriately high concentrations (typical ratios of 1:1000 sam- ple:matrix), concentrations which are necessary to achieve good signal resolution during the desorption/ ionization analysis; hence the matrix has been added after soft-landing. After digestion, the resulting mass spectrum Figure 7 shows 10 different tryptic fragments of cytochrome $c$, which corresponds to a calculated sequence coverage of $82 \%$. Besides cytochrome $c$ fragments, autolysis products of trypsin, various glycerol or fructose adducts, and a few lysozyme fragments (corresponding to less than $5 \%$ of the overall intensity; these are ascribed to contributions from the +17 ion, $m / z$ 843, which was incompletely separated) were also observed. 
Table 1. Peptides from tryptic digestion of soft-landed cytochrome $c$

\begin{tabular}{|c|c|c|c|c|c|c|c|c|c|c|}
\hline $\begin{array}{l}\text { MOWSE } \\
\text { score }\end{array}$ & $\begin{array}{l}\# / 21(\%) \\
\text { masses } \\
\text { matched }\end{array}$ & $\%$ Cov & $\begin{array}{c}\% \\
\mathrm{TIC}\end{array}$ & $\begin{array}{c}\text { Mean } \\
\text { err } \\
\text { ppm }\end{array}$ & $\begin{array}{l}\text { Data tol } \\
\text { ppm }\end{array}$ & $\begin{array}{c}\text { MS- } \\
\text { digest } \\
\text { index } \\
\#\end{array}$ & $\begin{array}{l}\text { Protein MW } \\
\text { (Da)/pl }\end{array}$ & $\begin{array}{c}\text { Accession } \\
\text { no. }\end{array}$ & Species & Protein name \\
\hline 1 & 10210 & $21(100)$ & 100 & 100 & 0.00378 & 0.0483 & $11572 / 9.5$ & P00006 & BOVIN & Cytochrome $c$ \\
\hline 2 & 3657 & $15(71)$ & 83 & 71.4 & 0.00054 & 0.0522 & $11486 / 9.5$ & P00012 & MIRLE & Cytochrome $c$ \\
\hline 3 & 3652 & $15(71)$ & 83 & 71.4 & 0.00054 & 0.0522 & $11501 / 9.6$ & $P 00011$ & CANFA & Cytochrome $c$ \\
\hline 4 & 735 & $14(66)$ & 74 & 66.7 & 0.00639 & 0.05 & $11516 / 9.6$ & P00010 & ESCGI & Cytochrome $c$ \\
\hline 5 & 734 & $14(66)$ & 74 & 66.7 & 0.00639 & 0.05 & $11530 / 9.5$ & P00007 & HIPAM & Cytochrome $c$ \\
\hline 6 & 301 & $11(52)$ & 58 & 52.4 & 0.0054 & 0.0538 & $11476 / 9.5$ & P00022 & CHESE & Cytochrome $c$ \\
\hline 7 & 300 & $11(52)$ & 58 & 52.4 & 0.0054 & 0.0538 & $11493 / 9.5$ & P81280 & ALLMI & Cytochrome $c$ \\
\hline 8 & 169 & $12(57)$ & 61 & 57.1 & 0.00907 & 0.0531 & $11560 / 9.5$ & P00008 & RABIT & Cytochrome $c$ \\
\hline 9 & 16.5 & $10(47)$ & 66 & 47.6 & -3.93 & 24.9 & $11456 / 9.5$ & $P 00023$ & CROAT & Cytochrome $c$ \\
\hline
\end{tabular}

aMS-FIT results from a computerized search, using the peptides observed in the tryptic digestion of soft-landed cytochrome $c$ as input parameters, correctly identify the soft-landed material as cytochrome $c$ (Bos taurus).

The peptide $m / z$ values were entered into MS-FIT [44], a peptide database which performs theoretical digestions on the set of proteins contained in the Swiss-Prot database. Among the most probable matches for the tryptic peptides entered, cytochrome $c$ Bos Taurus had the highest score (Table 1) allowing its identification as the soft-landed material.

One can envision the use of this method in a case where a native mixture of unknown proteins is separated by ion soft-landing and deposited into an array of enzymatically active liquids, at predetermined positions. Upon digestion and MALDI analysis of each individual spot and with the use of a search algorithm such as MS-FIT, the unknown proteins could be identified.

\section{Conclusions}

In this paper, we have demonstrated that proteins can survive the electrospray ionization and vacuum deposition process, and, from a qualitative point of view, do so without loss in their native properties. Moreover, the prospect of separating proteins from mixtures using ion soft-landing and depositing them into liquid surfaces has been demonstrated. Such liquid surfaces can be used for direct analysis by MALDI-MS or they can be further processed to exploit the bioactivity of the separated and softlanded chemical species. In the case of functionalized enzymatic surfaces, the current study has demonstrated that an enzyme substrate can be soft-landed and digested so that the nature of the soft-landed species can be determined by standard enzymatic tests, e.g., as in the case discussed, through the use of peptide identification and peptide search algorithms. One significant result is the demonstration, in the case of lysozyme, that soft-landing of the multiplyprotonated protein ion onto a fluorinated selfassembled monolayer surface occurs with incomplete neutralization. This allows accumulation of multiplycharged ions on the F-SAM surface and their subsequent desorption from the surface by MALDI. This experiment provides ions of lower mass/charge ratios of these high mass ions, just as is seen in ESI spectra. An important implication of these experiments is that it should be straightforward to perform soft-landing and product material analysis in the same device, without removing the soft-landed material from vacuum.

Further studies with other proteins having different $\mathrm{pI}$, stability and conformational properties are planned to continue to evaluate this use of soft-landing into active liquid surfaces as a possible new technology to separate and identify unknown biological species from complex mixtures. The storage of ions as a means of protein immobilization clearly is a topic deserving close scrutiny.

\section{Acknowledgments}

This work was supported by Inproteo Inc. (Indianapolis, Indiana) and Prosolia Inc. (Indianapolis, Indiana). The authors acknowledge helpful discussions with Richard DiMarchi, John Campbell, John Hale, John Hurrell, Michael Knierman, Rick Ludwig, Andy Guymon, and Peng Pan.

\section{References}

1. Franchetti, V.; Solka, B. H.; Baitinger, W. E.; Amy, J. W.; Cooks, R. G. Soft-Landing of Ions as a Means of Surface Modification. Int. J. Mass Spectrom. Ion Processes 1977, 23, 29-35.

2. Miller, S. A.; Luo, H.; Pachuta, S. J.; Cooks, R. G. Soft-Landing of Polyatomic Ions at Fluorinated Self-Assembled Monolayer Surfaces. Science 1997, 275, 1447-1450.

3. Tsekouras, A. A.; Iedema, M. J.; Cowin, J. P. Amorphous Water-Ice Relaxations Measured with Soft-Landed Ions. Phys. Rev. Lett. 1998, 80, 5798-5801.

4. Tsekouras, A. A.; Iedema, M. J.; Ellison, G. B.; Cowin, J. P. Soft-Landed Ions: A Route to Ionic Solution Studies. Int. J. Mass Spectrom. 1998, 174, 219-230.

5. Ouyang, Z.; Takats, Z.; Blake, T. A.; Gologan, B.; Guymon, A. J.; Wiseman, J. M.; Oliver, J. C.; Davisson, V. J.; Cooks, R. G. Preparing Protein Microarrays by Soft-Landing of Mass-Selected Ions. Science 2003, 301, 1351-1354.

6. Mayer, P. S.; Turecek, F.; Lee, H.; Scheidemann, A. A.; Olney, T. A.; Schumacher, F.; Strop, P.; Smrcina, M.; Patek, M.; 
Schirlin, D. 52nd ASMS Conference on Mass Spectrometry and Allied Topics. Nashville, Tennessee, 2004.

7. Luo, H.; Miller, S. A.; Cooks, R. G.; Pachuta, S. J. Soft-Landing of Polyatomic Ions for Selective Modification of Fluorinated Self-Assembled Monolayer Surfaces. Int. J. Mass Spectrom. 1998, 174, 193-217.

8. Geiger, R. J.; Melnyk, M. C.; Busch, K. L.; Bartlett, M. G. Modifications to an Analytical Mass Spectrometer for the SoftLanding Experiment. Int. J. Mass Spectrom. 1999, 183, 415-422.

9. Bromann, K.; Felix, C.; Brune, H.; Harbich, W.; Monot, R.; Buttet, J.; Kern, K. Controlled Deposition of Size-Selected Silver Nanoclusters. Science 1996, 274, 956-958.

10. Kitching, K. J.; Lee, H. N.; Elam, W. T.; Johnston, E. E.; MacGregor, H.; Miller, R. J.; Turecek, F.; Ratner, B. D. Development of an Electrospray Approach to Deposit Complex Molecules on Plasma Modified Surfaces. Rev. Sci. Instrum. 2003, 74, 4832-4839.

11. Feng, B. B.; Wunschel, D. S.; Masselon, C. D.; Pasa-Tolic, L.; Smith, R. D. Retrieval of DNA Using Soft-Landing After Mass Analysis by ESI-FTICR for Enzymatic Manipulation. J. Am. Chem. Soc. 1999, 121, 8961-8962.

12. Fuerstenau, S. D.; Benner, W. H.; Thomas, J. J.; Brugidou, C.; Bothner, B.; Siuzdak, G. Mass Spectrometry of an Intact Virus. Angewandte Chemie Int. Ed. 2001, 40, 542-544.

13. Trauger, S. A.; Junker, T.; Siuzdak, G. Investigating Viral Proteins and Intact Viruses with Mass Spectrometry. Modern Mass Spectrom. 2003, 225, 265-282.

14. Fenselau, C.; Demirev, P. A. Characterization of Intact Microorganisms by MALDI Mass Spectrometry. Mass Spectrom. Rev. 2001, 20, 157-171.

15. Back, J. F.; Oakenfull, D.; Smith, M. B. Increased ThermalStability of Proteins in the Presence of Sugars and Polyols. Biochemistry 1979, 18, 5191-5196.

16. Davis-Searles, P. R.; Saunders, A. J.; Erie, D. A.; Winzor, D. J.; Pielak, G. J. Interpreting the Effects of Small Uncharged Solutes on Protein-Folding Equilibria. Annu. Rev. Biophys. Biomol. Struct. 2001, 30, 271-306.

17. Lakshmi, T. S.; Nandi, P. K. Effects of Sugar Solutions on Activity-Coefficients of Aromatic Amino-Acids and Their NAcetyl Ethyl-Esters. J. Phys. Chem. 1976, 80, 429-452.

18. Wimmer, R.; Olsson, M.; Petersen, M. T. N.; HattiKaul, R.; Petersen, S. B.; Muller, N. Towards a Molecular Level Understanding of Protein Stabilization: The Interaction Between Lysozyme and Sorbitol. J. Biotechnol. 1997, 55, 85-100.

19. Yu, Z. H.; Li, B. The Effect of Polyols on the Reactivation of Guanidium Chloride-Denatured Arginine Kinase from Shrimp Feneropenaeus chinensis Muscle. Prot. Pept. Lett. 2003, 10, 199-211.

20. Glish, G. L.; Todd, P. J.; Busch, K. L.; Cooks, R. G. MS MS Spectra of Organic Ions Generated by Secondary Ion Mass-Spectrometry. Int. J. Mass Spectrom. Ion Processes 1984, 56, 177-192.

21. Barber, M.; Bordoli, R. S.; Sedgwick, R. D.; Tyler, A. N. Fast Atom Bombardment of Solids (Fab) - A New Ion-Source for MassSpectrometry. J. Chem. Soc. Chem. Commun. 1981, 325-327.

22. Fenselau, C.; Cotter, R. J. Chemical Aspects of Fast-AtomBombardment. Chem. Rev. 1987, 87, 501-512.

23. Pachuta, S. J.; Cooks, R. G. Mechanisms in Molecular Sims. Chem. Rev. 1987, 87, 647-669.

24. Caprioli, R. M.; Suter, M. J. F. Continuous-Flow Fast-AtomBombardment-Recent Advances and Applications. Int. J. Mass Spectrom. Ion Processes 1992, 118, 449-476.

25. Seifert, W. E.; Caprioli, R. M. Fast Atom Bombardment Mass Spectrometry. In High Resolution Separation and Analysis of Biological Macromolecules, Karger, B. L., Hancock, W. S.; Academic Press: San Diego, CA, 1996; p453-486.
26. Karas, M.; Hillenkamp, F. Laser Desorption Ionization of Proteins with Molecular Masses Exceeding 10,000 Daltons. Anal. Chem. 1988, 60, 2299-2301.

27. Tanaka, K.; Waki, H.; Ido, Y.; Akita, S.; Yoshida, Y.; Yoshida, T. Protein and Polymer Analyses Up to $m / z$ 100,000 by Laser Ionization Time of Flight Mass Spectrometry. Rapid Commun. Mass Spectrom. 1988, 2, 151-153.

28. Williams, T. L.; Fenselau, C. p-Nitroaniline/Glycerol: A Binary Liquid Matrix for Matrix-Assisted Laser Desorption/ Ionization Analysis. Eur. Mass Spectrom. 1998, 4, 379-383.

29. Ho, Y. P.; Fenselau, C. Applications of $1.06 \mu \mathrm{m}$ IR Laser Desorption on a Fourier Transform Mass Spectrometer. Anal. Chem. 1998, 70, 4890-4895.

30. Iavarone, A. T.; Williams, E. R. Mechanism of Charging and Supercharging Molecules in Electrospray Ionization. J. Am. Chem. Soc. 2003, 125, 2319-2327.

31. Iavarone, A. T.; Williams, E. R. Supercharging in Electrospray Ionization: Effects on Signal and Charge. Int. J. Mass Spectrom. $2002,219,63-72$.

32. Iavarone, A. T.; Jurchen, J. C.; Williams, E. R. Supercharged Protein and Peptide Clone Formed by Electrospray Ionization. Anal. Chem. 2001, 73, 1455-1460.

33. Takáts, Z.; Wiseman, J. M.; Gologan, B.; Cooks, R. G. Electrosonic Spray Ionization. A Gentle Technique for Generating Folded Proteins and Protein Complexes in the Gas Phase and for Studying Ion-Molecule Reactions at Atmospheric Pressure. Anal. Chem. 2004, 76, 4050-4058.

34. Sze, E. T. P.; Chan, T. W. D.; Wang, G. Formulation of Matrix Solutions for Use in Matrix-Assisted Laser Desorption Ionization of Biomolecules. J. Am. Soc. Mass Spectrom. 1998, 9, 166-174.

35. Russell, W. K.; Russell, D. H.; Busby, M. B.; Kolberg, A.; Li, S.; Maynard, D. K.; Sanchez-Vindas, S.; Zhu, W.; Vigh, G. Artifact-Free Matrix-Assisted Laser Desorption Ionization Timeof-Flight Mass Spectra of Tert.-Butyldimethylsilyl Ether Derivatives of Cyclodextrins Used for the Synthesis of SingleIsomer, Chiral Resolving Agents for Capillary Electrophoresis. J. Chromatogr. A 2001, 914, 325-330.

36. Murray, K. K.; Russell, D. H. Liquid Sample Introduction for Matrix-Assisted Laser-Desorption Ionization. Anal. Chem. 1993, 65, 2534-2537.

37. Blake, T. A.; Ouyang Z.; Wiseman, J. M.; Takáts, Z.; Guymon, A. J.; Kothari, A.; Cooks, R. G. Preparative Linear Ion Trap Mass Spectrometer for the Collection of Purified Proteins and Peptides into Arrays Using Ion Soft-Landing; unpublished.

38. Wiseman, J. M.; Takáts, Z.; Gologan, B.; Davison, V. J.; Cooks, R. G. Direct Characterization of Enzyme-Substrate Complexes Using Electrosonic Spray Ionization Mass Spectrometry. Angewandte chemie, manuscript submitted.

39. Chorush, R. A.; Little, D. P.; Beu, S. C.; Wood, T. D.; Mclafferty, F. W. Surface-Induced Dissociation of Multiply Protonated Proteins. Anal. Chem. 1995, 67, 1042-1046.

40. Gao, H.; Leary, J. A. Multiplex Inhibitor Screening and Kinetic Constant Determinations for Yeast Hexokinase Using Mass Spectrometry Based Assays. J. Am. Soc. Mass Spectrom. 2003, 14, 173-181.

41. Alvarez, J.; Laskin, J.; Cooks, R. G. Soft-landing of peptides by mass spectrometry; unpublished.

42. Dale, M. J.; Knochenmuss, R.; Zenobi, R. Graphite/Liquid Mixed Matrices for Laser Desorption/Ionization Mass Spectrometry. Anal. Chem. 1996, 68, 3321-3329.

43. Cornett, D. S.; Duncan, M. A.; Amster, I. J. Liquid-Mixtures for Matrix-Assisted Laser-Desorption. Anal. Chem. 1993, 65, 26082613.

44. Clauser, K. R.; Baker, P.; Burlingame, A. L. Role of Accurate Mass Measurement $( \pm 10 \mathrm{ppm})$ in Protein Identification Strategies Employing MS or MS MS and Database Searching. Anal. Chem. 1999, 71, 2871-2882. 